\title{
DIE SÄULE DES MARCUS AURELIUS: NARRATIVE STRUKTUR UND IDEOLOGISCHE BotschafT TONIO HÖlSCHER, Heidelberg*
}

\section{Einführung}

Die Säule des Marc Aurel imitiert die Säule des Traian ${ }^{1}$. Da die Traians-Säule bis zur Zeit des Marc Aurel ein einzigartiges Monument war, ist die Angleichung zweifellos die erste und wichtigste Botschaft gewesen: Marc Aurel und seine Kriege sollten genau so groß wie Traian und seine Siege erscheinen.

Die Archäologen interessieren sich aber mehr für die Unterschiede. Sie heben hervor, in welcher Weise die Säule des Marc Aurel von der des Traian abweicht ${ }^{2}$. Gleichheit und historische Statik sind Phänomene, denen die Historiker grundsätzlich nicht viel abgewinnen: Historische Methoden zielen in erster Linie auf Diversität, Veränderung, Individualität ${ }^{3}$. Das ist wissenschaftlich durchaus legitim: Der Historiker hat die freie Wahl, auf welche Aspekte der Vergangenheit er den Blick richtet.

Aber: Historische Gesellschaften und ihre Kulturen sind grundsätzlich auf den verschiedenen Sektoren des Lebens zugleich von Veränderungen ergriffen und von Konstanz geprägt. Dynamische Impulse stehen neben statischen Faktoren; plötzlich einschneidende Ereignisse neben langfristig verlaufenden Prozessen und festen gewachsenen Strukturen; jähe Wechsel, die bewußt erlebt werden, aber an der Oberfläche bleiben, neben kumulativem Wandel, der sich unbewußt vollzieht und von den Zeitgenossen erst später oder auch gar nicht bemerkt wird. Historische Dynamik wird erst sinnvoll erfaßbar, wenn sie in ihre Komponenten zerlegt wird: in die gesellschaftlichen und politischen, religiösen, kulturellen und mentalen Sektoren, mit ihren verschiedenen Stufen zwischen Bewußt- und Unbewußtheit, ihren spezifischen Tempi der Veränderung; und wenn diese Komponenten zu den Faktoren der Retardierung und der Statik ins Verhältnis gesetzt werden.

Eine Grundthese der folgenden Überlegungen ist: Die narrative Grundstruktur und das politische Grundkonzept der Marcus-Säule sind ähnlich wie bei der Traians-

* Die Reliefs der beiden Säulen werden in der üblichen Zählung der Szenen nach CichoRIUS (Bezeichnung: Ts) und PeTERSEN - v. DOMASZEWSKI - CALDERINI (Bezeichnung: Ms) zitiert. Dabei werden allerdings durchgängig arabische Zahlen verwendet. Mit diesen sind also bei der Traians-Säule die ,Einzelbilder“ (hier „Szenen“ genannt), nicht die bei Cichorius mit arabischen Zahlen bezeichneten Platten der Gipsabgüsse gemeint. Für Hilfe bei der Kontrolle der Münzprägungen danke ich Susanne Muth.

1 M. JoRDAN-Ruwe, Das Säulenmonument. Asia Minor Studien 19 (Bonn 1995) 84-91.

${ }^{2}$ Wichtig in dieser Hinsicht besonders M. WEGNER, Die kunsthistorische Stellung der Marcussäule. Jahrbuch des Deutschen Archäologischen Instituts 46 (1931) 61-174 (enthält mehr über Bildthemen und ideologische Bedeutung, als der Titel erwarten läßt). P.G. HAMBERG, Studies in Roman Imperial Art (Upsala 1945) 104-161.

${ }^{3} \mathrm{Zu}$ statischen Aspekten der römischen Repräsentationskunst und Politik: T. HŌLSCHER, Die Geschichtsauffassung in der römischen Repräsentationskunst. Jahrbuch des Deutschen Archäologischen Instituts 95 (1980) 313-317. 
Säule — aber der mentale und psychische Habitus und, damit zusammenhängend, die stilistischen Formen haben sich stark verändert. Das bedeutet: Der Blick wird sich zunächst auf die expliziten politischen Themen und Botschaften der Reliefs richten, die die konkrete Funktion dieses Denkmals bildeten. Er wird aber dabei nicht stehen bleiben, sondern dahinter nach Phänomenen der kollektiven Psychologie und Mentalität fragen. Es wird nicht überraschen, daß am Ende beides, Bildthemen und Stil, Programm und Psychologie, in enger Wechselwirkung zueinander steht.

\section{Sichtbarkeit und Bedeutung}

Eine grundsätzliche Voraussetzung beider Säulen ist ihre Sichtbarkeit. Zu der Traians-Säule hat sich über diese Frage eine intensive Diskussion entwickelt ${ }^{4}$. Das Paradox liegt darin, daß die Reliefs der Säule nach einem sehr komplexen Konzept organisiert sind, daß sie auch in der Komposition und den Stilformen von hohem Rang sind, daß dies alles aber für die Betrachter am Fuß der Säule kaum wahrzunehmen ist. Dies Problem ist nicht dadurch aufzulösen, daß man den Römern der Kaiserzeit bessere Augen attestiert, oder daß man sie zum Betrachten der Reliefs auf die Dächer der umliegenden Gebäude steigen läßt: Das Paradox bleibt bestehen und muß von der Forschung akzeptiert werden. Paul Veyne hat daraus die Forderung abgeleitet, daß die Archäologen sich auf die Sichtbedingungen der antiken Betrachter einstellen und von ihren hochkomplexen, hypertrophen Interpretationen Abstand nehmen müssen ${ }^{5}$. Daran ist der erste Teil zweifellos berechtigt: Als Historiker wird man zunächst die konkrete Wirkung auf das antike Publikum rekonstruieren. Der zweite Teil der Forderung dagegen ist zweifellos falsch, denn er beruht auf einem grundsätzlich irrigen Konzept des antiken Bildwerks: Vor der Epoche des Museums und der intensiven Betrachtung von ,Kunst' besaß jedes Bildwerk weit mehr Aspekte, als im konkreten „,Gebrauch“ wahrgenommen wurden.

In Begriffen der Semiotik kann man bei den beiden Säulen, wie bei vielen anderen antiken Staatsdenkmälern, von „Botschaften mit reduzierter Information“ sprechen 6 . Das besagt, daß Botschaften von Monumenten in der normalen Situation des Lebens grundsätzlich nur partiell wahrgenommen werden und trotzdem ihre Wirkung ausüben; daß sie aber, um diese Wirkung auszuüben, vollständig durchgestaltet sein müssen. Der Betrachter der Säule kann nicht alle Teile erkennen, und kann auch nicht das Konzept in seiner ganzen Komplexität durchschauen; aber wenn er die untersten Szenen betrachtet oder die Säule aus der Distanz sieht, muß er sicher sein, daß die Kriege bis oben hin in der gleichen Detailliertheit geschildert werden, und daß die Bilderfolge im ganzen nach einem ideologischen Konzept organisiert ist. Ohne ,potentielle Kontrolle“, auch wenn sie fiktiv bleibt, kann kein Bildwerk Wirkung aus-

${ }^{4} \mathrm{Zu}$ den älteren Ansätzen s. T. Hölscher, in: L. BAumer - T. HÖlscher - L. WinKLer, Narrative Systematik und politisches Konzept in den Reliefs der Traianssäule. Jahrbuch des Deutschen Archäologischen Instituts 106 (1991) 262.

${ }^{5}$ P. VEYNE, Conduites sans croyance et œvres d'art sans spectateurs. Diogène 143 (1988) 2 22.

6 T. Hölscher, in: L. Baumer - T. HÖlscher - L. Winkler, a.O. (Anm. 4) 262-264. Ausführlicher S. SETtis, Die Trajanssäule: Der Kaiser und sein Publikum. In: J. ArrouYE u.a. (Hg.), Die Lesbarkeit der Kunst. Zur Geistes-Gegenwart der Ikonologie (Berlin 1992) 40-52. 
üben. Die Wirkung besteht darin, daß der Betrachter, auch wenn er nur flüchtig oder aus der Distanz auf das Monument blickt, doch so viel begreift, daß hier die Kriege des Kaisers in größter Ausführlichkeit und in rühmenswertester Weise geschildert sind. Er kann sich an den blicknahen, gut sichtbaren unteren Windungen der Säule vergewissern, in welcher dokumentarisch-panegyrischen Weise die Kriege Traians bzw. Marc Aurels verherrlicht werden; und er kann trotz allmählich nachlassender Erkennbarkeit eine Vorstellung gewinnen, daß dies bis in die symbolische Höhe von $100 \mathrm{Fuß}$ so weiter geht. Auch wenn er dies nicht im einzelnen bis oben nachvollziehen kann, partizipiert er an einem allgemeinen ideologischen Diskurs, der in den Monumenten geführt wird und der die Wahrnehmung des einzelnen Betrachters transzendiert. Im Grund gilt das für alle kulturellen Botschaften, sogar bis hin zu den neuzeitlichen Werken der „Kunst“: Das Potential der Bildwerke ist immer größer, als es vom einzelnen Betrachter realisiert wird.

\section{Grund-Strukturen und Veränderungen}

Die Säulen des Traian und des Marc Aurel besitzen die gleiche Grundstruktur, die aus folgenden Elementen besteht:

- Die Form des Monuments, mit Sockel, columna centenaria und Statue des Kaisers;

- Der Schmuck des Säulenschaftes mit einem Spiralband, auf dem in Reliefs die Kriegstaten des Kaisers geschildert werden;

- Die Organisation des Reliefbandes: kontinuierende Darstellung der Kriegszüge in einer Sequenz, die als zeitliche Kontinuität gelesen werden soll; Teilung in zwei Hälften durch Victoria zwischen zwei Tropaea;

- Die Form der Darstellung mit militärischen Aktionen in landschaftlichem Raum;

- Die Hervorhebung bestimmter exemplarischer Standard-Szenen von ideologischer Bedeutung, vor allem zu Beginn der Feldzüge, in mehr oder minder festgelegter Folge.

Diese Elemente haben die Betrachter der Marcus-Säule zweifellos als intentionale Nachahmung der Traians-Säule wahrnehmen können und wohl auch tatsächlich wahrgenommen.

Innerhalb dieser Grundstruktur aber werden starke Veränderungen deutlich. In der Forschung, besonders in Deutschland, wurden vor allem Veränderungen der künstlerischen Form, der sog. „Stilwandel“ der spätantoninischen Kunst, hervorgehoben. Dessen Interpretation ging in verschiedene Richtungen. In der geistesgeschichtlichen Epoche seit den 20er Jahren sah man hier einen zunächst formalen Schritt in Richtung auf die Spätantike, der dann aber als Symptom für geistige Tendenzen, für spirituelle Weltanschauungen, Krisenängste etc. gewertet wurde ${ }^{7}$. Dagegen hat F. Pirson kürzlich

${ }^{7}$ Der ,antoninische Stilwandel“ wäre ein interessanter Gegenstand archäologischer Forschungsgeschichte. Wichtigste Positionen: G. RoDENWALDT, Über den Stilwandel in der antoninischen Kunst. Abhandłungen der Preußischen Akademie der Wissenschaften, phil.-hist. Klasse 1935 Nr. 3. P.G. Hamberg, Studies a.O. (Anm. 2) 178-181. O. PelikÁn, Vom antiken Realismus zur spätantiken Expressivität (Praha 1965) 29-67. Ders., Übergangs- und Krisenperioden in 
in bemerkenswerter Weise die Stilformen der Marcus-Säule mit deren spezifischer Botschaft verbunden und sie aus der Absicht einer plakativen Deutlichkeit erklärt ${ }^{8}$.

Weniger beachtet wurde dagegen die narrative Struktur der Bilderfolge. Neuere Untersuchungen haben an der Traians-Säule gezeigt, daß deren narrative Struktur nicht nur eine formale Strategie ist, sondern die ideologischen Botschaften bis in die Einzelheiten prägt ${ }^{9}$. Dasselbe gilt für die Marcus-Säule: Hier folgen die narrativen Sequenzen veränderten Prinzipien, und entsprechend verändert erscheint die ideologische Aussage. Auf dieser Grundlage ergibt sich auch eine neue Perspektive auf die Stilformen.

Zum Kontrast ist zunächst ein Blick auf die narrative Organisation der TraiansSäule nötig.

\section{Traians-Säule: Grundzüge der narrativen Struktur}

An der Traians-Säule wird der Bildbericht über die beiden Kriege gegen die Daker einem rigorosen Schema unterworfen, das zu einer höchst rationalen Typisierung der Handlungssequenzen führt. Grundlegend ist die Trennung in die zwei Kriege 101-102 und 105-106 n.Chr., und innerhalb dieser zwei Hälften die Unterscheidung in offensive und defensive Feldzüge: Der erste römische Angriff im Sommer $101 \mathrm{n}$.Chr. (1. Feldzug) wird in Szene Ts 1-30 geschildert, der dakische Gegenangriff und seine Abwehr im Winter 101-2 n.Chr. (2. Feldzug) in Szene Ts 31-45, der zweite Angriff der Römer im Sommer 102 n.Chr. (3. Feldzug) in Szene Ts 46-77. In der oberen Hälfte folgt die Wiederaufnahme der Kämpfe nach dem dakischen Aufstand im Winter 105-6 n.Chr. (4. Feldzug) in Szene Ts 78-100, und schließlich die dritte Offensive von 106 n.Chr. (5. Feldzug) in Szene Ts 101-155.

Die drei offensiven Kampagnen werden durchweg mit einem regulären Einleitungs-Zeremoniell eröffnet: Profectio - Lustratio - Adlocutio (Ts 3/5 - 8 - 10, 48/50 - 53 - 54, 101/2 - 103 - 104); zu Beginn der beiden Kriege, also im 1. und 5. Feldzug, ist eine Szene des Kriegsrates hinzugefügt (Ts 6, 105). In diesen Sequenzen werden die ideologischen Grundlagen römischer Kriegsführung rituell zur Wirkung gebracht: entschlossene virtus in der Profectio, pietas und providentia in der Lustratio, concordia und fides in der Adlocutio, consilium in der Beratung. Danach folgt der Vormarsch mit dem Bau von Festungen und Straßen, als materielle, d.h. technische und logistische Grundlage des römischen Erfolgs (Ts 11-20, 55/56); diese Szenen sind zugleich eine ideologische Dokumentation der Bereitschaft zum Ertragen von

der antiken Kunst (Brno 1977) 56-64. R. BIANCHI BANDINELLI, Rom, Das Zentrum der Macht (München 1970) 309-339, bes. 319-320. B. ANDREAE, Römische Kunst (1973) 247-269. H. JUNG, Zur Vorgeschichte des spätantoninischen Stilwandels. Marburger WinckelmannProgramm 1984, 59-103. Diskussion bei K. STROBEL, Das Imperium Romanum im ,3. Jahrhundert'. Modell einer historischen Krise? (Stuttgart 1993) 39-48.

${ }^{8}$ F. PIRSON, Style and Message on the Column of Marcus Aurelius. Papers of the British School at Rome 64 (1996) 139-179.

${ }^{9}$ Hierzu und zum Folgenden W. GAUER, Untersuchungen zur Trajanssäule (Berlin 1977). T. HÖLSCHER, Die Geschichtsauffassung a.O. (Anm. 3) 290-297. L. BAUMER - T.HÖLSCHER - L. WINKLER, a.O. (Anm. 4). S. SETTIS, La colonne Trajane: invention, composition, disposition. Annales, Économies sociétés civilisations 40 (1985) 1151-1194. Ders. (Hg.), La colonna Traiana (Torino 1988) 86-255. 
Strapazen, von labor. Dabei werden dem Kaiser bereits die ersten Gefangenen vorgeführt, als Zeichen der vigilantia der Soldaten (Ts 18, 52). Schließlich führt ein rascher Aufbruch zu einer oder mehreren siegreichen Schlachten, gefolgt von einer Adlocutio zur Belobigung der Soldaten und einer Unterwerfung weiterer Gegner (Ts 21-28, 57 75, 106-126). Den Schluß bilden Schilderungen der katastrophalen Kriegsfolgen für die Daker (Ts 29/30, 76, 142-155).

Von diesem Schema weichen die beiden defensiven Feldzüge darin ab, daß das einleitende Zeremoniell fehlt. Stattdessen wird die Störung des Friedens durch die Daker geschildert (Ts 32, 93/94). Die Römer reagieren darauf durch unmittelbares Vordringen, das wiederum zu einem entscheidenden Sieg (Ts 40/41, 95/97) führt.

Innerhalb dieser stereotypen Folgen von Szenen werden Züge der militärischen Aktionen wiedergegeben, die der spezifischen Schilderung der einzelnen Feldzüge dienen. Man könnte das so verstehen, daß die narrativen Grundschemata zugrunde gelegt und dann mit Aspekten der Realität angereichert wurden. Plausibler ist wohl die umgekehrte Vorstellung, daß die Erfahrung der realen Kriegszüge zugrunde lag und dann in dem Relieffries nach dem Muster der narrativen Grundschemata begriffen, geordnet und dargestellt wurde.

Neben der realistischen Entfaltung durch Aspekte der historischen Wirklichkeit steht aber eine höchst kalkulierte konzeptionelle Differenzierung der Grundschemata untereinander, die dem römischen Sieg eine ideologische Dynamik und emphatische Glorifizierung vermitteln soll ${ }^{10}$ :

- Die drei Szenen der Profectio erscheinen, vom 1. über den 3. bis zum 5. Feldzug, in einer stufenweisen Steigerung, die den Kaiser immer stärker aus dem Heer heraushebt und zum Gegenstand von Ruhm und Verehrung macht (Ts 3/5, 48/50, 101/2).

- Bei der Adlocutio wird genau zwischen aufmunternden Reden zum Beginn der Feldzüge, Lobreden nach siegreichen Schlachten und Abschlußreden am Ende der Feldzüge unterschieden.

- Der Bau von Festungen wird vor allem im 1. Feldzug breit geschildert, um die technischen Grundlagen des römischen Sieges zu betonen; nur hier werden diese Aktivitäten durch die Gegenwart des Kaisers aufgewertet (Ts 11-20).

- Der Vormarsch der Truppen dagegen wird vom 1. bis zum 5. Feldzug immer ausführlicher gezeigt: Zuletzt hat er den Charakter einer Heeresschau, einer Demonstration aller Truppen, die einem unaufhaltsamen Sieg entgegen gehen (Ts 21/22, 57/59, 106/9).

- Bei den entscheidenden Schlachten ist nur im 1. Feldzug Iuppiter selbst anwesend, um die grundsätzliche Hilfe der Götter für die Römer anzuzeigen (Ts 24). Im 2. Feldzug werden zum einzigen Mal römische Verwundete gezeigt, um die starke Bedrohung durch die dakische Offensive anzuzeigen, zugleich aber auch um bei dieser Gelegenheit die gute medizinische Versorgung im römischen Heer zu bezeugen (Ts 40/41). Im 3. Feldzug dagegen ist die Zahl der Kämpfe auf drei Schlachten gesteigert, in denen ein System von überlegenen römischen Kampfweisen demonstriert wird: erst eine „Materialschlacht“" mit Geschützen (Ts

${ }^{10}$ Zum Folgenden s. L. BAUMER - T. HÖLSCHER - L. WinKLER, a.O. (Anm. 4). Adlocutio: 278287 (L.B.). Profectio, Festungen, Vormarsch, Schlachten: 287-295 (T.H.). Dazu J.M. DAVID in diesem Band pp. 213-226. 
66/67), dann eine taktische Schlacht mit Exempla römischer Disziplin und Koordination, gipfelnd im Angriff auf eine feindliche Festung in der Formation der testudo (Ts 70/71), zuletzt ein vollständiger römischer Sieg (Ts 72). Im 5. Feldzug folgt schließlich die endgültige, überwältigende Eroberung der dakischen Bergfestungen, die kaum einnehmbar scheinen (Ts 113-116).

- Sogar die anschließenden Unterwerfungen sind differenziert. Nur im 1. Feldzug erscheinen zwei Gruppen von Dakern vor dem Kaiser: zunächst eine Gesandtschaft, die offenbar kooperativ ist, die zu Fuß ankommt und von Traian gnädig empfangen wird (Ts 27); darauf offenbar eine renitente Gruppe, zu Pferd sitzend, die vom Kaiser gar nicht wahrgenommen wird (Ts 28). Hier, zu Beginn des Krieges, wird besonders unterschieden zwischen einsichtigen Dakern, die mit Nachsicht und Integration rechnen können, und den superbi, die die ganze Härte römischer Vergeltung zu spüren bekommen. Auch diese submissiones werden im weiteren Verlauf immer mehr gesteigert, bis zum hilflosen Kniefall eines einzelnen dakischen Fürsten zwischen dem Spalier römischer Legionäre im 5. Feldzug (Ts 118, s. auch 123).

Ähnliches trifft auch für alle anderen Szenen der Traians-Säule zu. Bei jeder einzelnen Szene ist zu erkennen, warum sie gerade an dieser Stelle und in dieser spezifischen Form dargestellt ist. Eine genau kalkulierte Strategie beherrscht das ganze Konzept. Jeder Feldzug bildet einen groß gespannten Bogen, in dem die ideologische und technische Superiorität der Römer in vielen Aspekten systematisch entfaltet wird. Und alle fünf Feldzüge bilden zusammen eine umfassende Struktur, bei der Offensive und Defensive, Anfang, Mitte und Ende dynamisch aufeinander bezogen sind.

\section{Marcus-Säule: Grundzüge der narrativen Struktur. Emphatisches Staccato}

An der Marcus-Säule ist diese systematische Gesamtstruktur zersetzt. Stattdessen stehen andere Qualitäten im Vordergrund. Eine strukturelle Lektüre der Reliefs läßt gegenüber der Traians-Säule Unterschiede im narrativen Rhythmus erkennen, die eine veränderte ideologische und mentale Grundhaltung bezeugen.

Auch an der Marcus-Säule ist das Reliefband in zwei große Teilkriege von je zwei Kriegsjahren unterteilt. Anscheinend wird das Kriegsjahr 172 n.Chr. in den Szenen Ms 1-29 [Fig. 1-47] geschildert, das Jahr 173 n.Chr. in den Szenen Ms 30-55 [Fig. 4875]; im oberen Teil umfaßt das Jahr 174 n.Chr. die Szenen Ms 56-77 [Fig. 76-96], das Jahr 175 n.Chr. die Szenen Ms 78-115 [Fig. 97-135].

Dabei ist aber auffallend, daß diese Zäsuren bei weitem nicht so deutlich sind wie an der Säule des Traian. Die Forschung hatte bei der Marcus-Säule viel größere Schwierigkeiten, thematische Abschnitte zu erkennen, und dies ist nicht ein Defizit der wissenschaftlichen Analyse, sondern eine Eigenschaft der Reliefs selbst. Der Grund dafür liegt in zwei Phänomenen, die sich gegenseitig ergänzen und verstärken.

- Die einzelnen Kriegsjahre werden nicht als jeweils ein einziger großer Handlungsbogen beschrieben, sondern zerfallen in mehrere kleinere Einheiten. Immer wieder wird der Aufbruch des Heeres geschildert, darauf folgt unmittelbar ein Sieg oder die Zerstörung einer Siedlung, und schließlich die Gefangennahme und Bestrafung der Gegner. Im 1. Kriegsjahr bilden anscheinend die Szenen Ms 1-9, 
10-21, 22-29 solche Einheiten, im 2. Kriegsjahr die Szenen Ms 30-43, 44-49, 5055. Und so weiter.

- Die zeremoniellen Szenen, die die Kriegszüge einleiten, mit Profectio, Lustratio, Adlocutio und Consilium, erscheinen an der Marcus-Säule weniger regelmäßig, weniger vollständig, weniger kompakt und weniger systematisch.

Die beiden Phänomene führen dazu, daß die Feldzüge des Marc Aurel nicht so stark von einem übergeordneten, systematischen und ideologischen Grundmuster geprägt sind. Dies ist die negative Seite des Befunds. Die eigentliche Begründung aber liegt darin, daß jetzt andere Botschaften in den Vordergrund treten. Auch sie haben ideologischen Charakter.

Die kleinen narrativen Einheiten haben einen typischen raschen Verlauf. Ein gutes Beispiel ist die Folge der Szenen Ms 50-55 [Fig. 72-75]: Vormarsch einer römischen Truppe (Ms 50), eindeutiger Sieg (Ms 50), die Gegner werden zu Verhandlungen gezwungen (Ms 51); darauf folgt ein weiterer römischer Angriff (Ms 52), der zur kniefälligen Unterwerfung der Gegner führt (Ms 53); schließlich wird die letzte Festung mit einer testudo erstürmt (Ms 54) und der Sieg mit einer Adlocutio gefeiert (Ms 55).

Ähnlich die Folge der Szenen Ms 56-62 [Fig. 77-79] zu Beginn des 3. Kriegsjahres: Der Kaiser empfängt anscheinend kooperative Barbaren im Lager (Ms 56), darauf brechen Reiter und Fußkämpfer auf und überfallen die Gegner im Wald (Ms 57); schon wenige Szenen später kommt es zur Hinrichtung der Anführer des Widerstands (Ms 61) und zur Unterwerfung der restlichen Gegner (Ms 62).

Worum es hier geht, ist deutlich: Entschlossener Angriff, rascher und totaler Sieg, harte Bestrafung. Besonders offensichtlich ist das in der nächsten Sequenz der Szenen Ms 63 ff. [Fig. 80 ff.], wo ein Staccato von Aufbruch und Vernichtung in Szene gesetzt wird: Die Reiter sprengen zusammen mit Fußkämpfern los und stechen sofort die ersten Gegner nieder (Ms 63); sie führen erbeutete Herden und gefangene Krieger ab (Ms 64-65) und präsentieren dem Kaiser abgeschlagene Köpfe (Ms 66). Der nächste Auszug der Legionäre führt sofort wieder zur Niederstechung der Gegner (Ms 6768 [Fig. 81-85]) und zur Vertreibung der verbliebenen Feinde (Ms 69 [Fig. 86-88]). Wenig später werden weitere Gegner verfolgt (Ms 72; Fig. 90), anschließend die Männer getötet und die Herden und Frauen weggeführt (Ms 73 [Fig. 90-91]).

Dies Konzept kennt keine Skrupel. Die Gegner, die sich gegen die Ordnung des Imperium Romanum erhoben haben, sind Verbrecher. Die härteste Strafe ist nur gerecht.

Die folgenden Beobachtungen und Bemerkungen sollen zeigen, wie stark dies Konzept die ganze Auffassung dieser Kriegszüge prägt.

\section{Sequenzen des Anfangs}

Die ideologische Begründung der römischen Überlegenheit tritt in diesem Konzept mehr in den Hintergrund. Bezeichnend ist der Anfang in den Szenen Ms 1-9 (Fig. 124). Die Gruppe der zeremoniellen Rituale zum Kriegsbeginn ist von der TraiansSäule übernommen, aber mit auffälligen Veränderungen. Nach der Profectio (Ms 3 [Fig. 11-13]) folgt gleich eine Adlocutio (Ms 4 [Fig. 14-15]): Die steigende Bedeutung des Heeres für die Stellung des römischen Kaisers hat hier zur Folge, daß die concordia mit den Soldaten am Anfang steht. Erst danach folgen pietas und providentia 
in der Lustratio (Ms 6 [Fig. 17-18]); die Szene des Kriegsrates als Zeichen von providentia und consilium wird sekundär, sie erscheint erst später und in sehr rudimentärer Form im nächsten Abschnitt des Krieges (Ms 14 [Fig. 31]).

Die weiteren Vorbereitungen des Krieges, der Bau von Festungen und Straßen, die an der Traians-Säule die ganze zweite Windung einnehmen, sind an der Marcus-Säule beim Beginn des Krieges völlig eliminiert — stattdessen stößt das Heer sofort gegen den Feind vor. Aber es kommt nicht einmal zu einer richtigen Schlacht: Die erste Siedlung ist bereits fluchtartig verlassen, die Häuser werden in Brand gesteckt, das Getreide wird in Besitz genommen (Ms 7 [Fig. 19-20]), gleich darauf werden zwei übergelaufene gegnerische Anführer vor den Kaiser geführt, dagegen werden die Flüchtlinge erstochen, andere liegen bereits tot am Boden (Ms 8 [Fig. 21-22]). Und schon wird der Sieg mit einer zweiten großen Adlocutio gefeiert (Ms 9 [Fig. 23-24]).

Von den weiteren Feldzügen wird das 2. und das 3. Kriegsjahr, obwohl auch sie als offensive Kampagnen geschildert sind, überhaupt nicht von einer Sequenz ideologischer Rituale eingeleitet. Erst das letzte Kriegsjahr beginnt wieder mit einer großen Profectio (Ms 78) und setzt mit Szenen der Beratung (Ms 80), des Lagerbaus (Ms 82) und der Adlocutio (Ms 83) fort. Aber der Kriegsrat und die Bauszene sind nur rudimentär ausgebildet; und die ganze Sequenz ist durchsetzt von militärischen Aktivitäten, daher besitzt sie keine starke ideologische Geschlossenheit.

\section{Rituelle Szenen}

Rituelle Szenen, in denen ideologische Leitbegriffe dargestellt werden, sind an der Marcus-Säule insgesamt selten. Sie werden allerdings durchaus bewußt an entscheidenden Stellen der Handlung eingesetzt.

Ein religiöses Opfer, Zeichen der pietas und der providentia, erscheint in der Form der Lustratio nur ein einziges Mal, sinnvollerweise zu Beginn des 1. Kriegsjahres (Ms 6 [Fig. 17]). Andere Opfer werden beim Aufbruch zu den nächsten Attacken, beim Überschreiten von Flüssen dargebracht (Ms 13 [Fig. 30-31]. Ms 30 [Fig. 47-49]): Der Anfang des ganzen Krieges wird unter den Schutz der Götter gestellt. Ein Dankopfer dagegen beschließt, statt einer Adlocutio, das 3. Kriegsjahr (Ms 75 [Fig. 93]): angemessen nach den besonders harten ,Arbeits"-Siegen, die in dieser Phase des Krieges errungen worden sind.

Dem entspricht, daß der Kriegsrat, als Demonstration von consilium, ebenfalls im ersten Teil des 1. Kriegsjahres (Ms 14 [Fig. 31]) und zu Beginn des letzten Feldzuges (Ms 80 ) erscheint, wenngleich in wenig hervorgehobener Bedeutung.

Stärker betont sind Szenen der Adlocutio: programmatisch gleich nach der Profectio zum 1. Feldzug (Ms 4 [Fig. 14-15]) und sofort wieder nach dem ersten Sieg (Ms 9); sodann zum Abschluß des 2. Kriegsjahres (Ms 55 [Fig. 75-76]) vor der Victoria. Das 3. Kriegsjahr mit seinen „Arbeits“-Siegen stellt dagegen im abschließenden Dankopfer die pietas heraus (Ms 75 [Fig. 93]); während im 4. Jahr die Soldaten vor den letzten großen Attacken die entscheidende Kampfmoral wieder in einer Ansprache des Kaisers zugesprochen bekommen (Ms 83 [Fig. 105], im Abschnitt 80-92, Ms 96 [Fig. 111-112], im Abschnitt 93-100). Und wie in der unteren Hälfte (Ms 55 [Fig. 75-76]) endet auch in der oberen Hälfte der endgültige Erfolg mit einer Sieges-Adlocutio (Ms 100 [Fig. 120-121]).

Nur im 4. Kriegsjahr werden schließlich zwei Szenen des Lagerbaues geschildert: wieder zu Beginn der beiden letzten großen Attacken (Ms 82 [Fig. 104]. Ms 94 [Fig. 
110]), in unmittelbarer Nähe der beiden Adlocutio-Szenen. Befestigte Lager erscheinen hier als Voraussetzung und zugleich als Steigerung des siegreichen Abschlusses des Krieges.

Alle diese Vorgänge, Profectio, Opfer, Kriegsrat, Adlocutio, Lagerbau, wurden zweifellos während der gesamten Feldzüge ständig vollzogen. In den Reliefs der Säule werden sie aber nur an wenigen Stellen hervorgehoben: dort, wo die ideologische Strategie der Darstellung es erforderte.

Im Konzept der Marcus-Säule ist also die Fähigkeit nicht verloren gegangen, rituelle Szenen zielgerichtet an entscheidenden Punkten der Handlung einzufügen und dadurch ideologische Akzente zu setzen.

Dennoch nehmen diese Szenen an der Marcus-Säule einen vergleichsweise reduzierten Raum ein. Die ideologischen Vorbereitungen der Feldzüge werden nicht mehr so systematisch vor Augen geführt, und die technischen Grundlagen der römischen Kriegsführung spielen kaum mehr eine Rolle. Dem entspricht, daß ein weiteres Thema an der Marcus-Säule völlig fehlt, das bei Traian extensiv in Szene gesetzt worden war: das hohe Niveau der römischen Zivilisation. An der Traians-Säule wird der Feldzug des Jahres 105 n.Chr., vor dem endgültigen Sieg, mit einer langen Sequenz von Szenen eingeleitet, in denen der Kaiser durch die Provinzen des Balkan zum Kriegsschauplatz zieht (Ts 81-91) ${ }^{11}$. Überall besucht er blühende Städte, mit Stadtmauern, Heiligtümern, Theatern; er wird von der Bevölkerung dieser Städte jubelnd empfangen, zelebriert feierliche Opfer und wird voller Ehrfurcht weiter geleitet. Hier werden die Segnungen der römischen Zivilisation gefeiert, die auf der concordia mit den Göttern und auf dem Konsens unter den Menschen beruht: eine weitere ideelle Grundlage der römischen Macht.

Bei Marc Aurel treten solche ideologischen Aspekte in den Hintergrund. Wahrscheinlich wurden sie nicht nur von den entwerfenden Bildhauern, sondern auch von dem Publikum dieser Zeit nicht mehr als vordringliches Thema angesehen. Der Krieg wurde nicht mehr so deutlich als komplexe Manifestation einer ganzen kulturellen Welt entfaltet, nicht mehr als Demonstration der ideologischen Konzepte, der technischen Grundlagen, der zivilisatorischen Leistungen, nicht mehr als Sieg eines Systems, das sich auf den Feldzügen in durchdachter Planung nach einem großen Grundmuster entfaltet. Sondern es geht um andere Aussagen und andere Akzente.

\section{Ideologie der Strafe}

Selbstverständlich ist auch die Marcus-Säule auf eine Ideologie hin orientiert. Aber diese Ideologie ist weniger komplex, weniger systematisch, weniger intellektuell konzipiert, sondern punktuell, faktisch - und emotional. Zugespitzt gesagt: Die Gegner Traians waren gewaltige historische Antipoden, die Gegner Marc Aurels sind verbrecherische Rebellen. Traian hatte einen großen welthistorischen Kampf geführt, Marc Aurel schlägt einen Aufstand nieder. Traian hatte eine große Macht mit äußerstem Einsatz bezwungen, Marc Aurel führt eine gerechte Strafe aus, in aller Härte und Brutalität. Dafür müssen nicht so sehr komplexe ideelle Konzepte entwickelt werden, sondern es wird effiziente Entschlossenheit demonstriert. Keine ideologische Be-

${ }^{11}$ Dazu L. Winkler, in: L. BAumer - T. HÖlscher - L. Winkler, a.O. (Anm. 4) 271-277. 
gründung, sondern kurzer Prozeß. Nicht konzeptionelle Legitimation, sondern emotionale Härte.

Diese Grundeinstellung prägt den narrativen Rhythmus der Marcus-Säule von Anfang bis Ende. Fast durchweg werden kurze Sequenzen von Vormarsch Vernichtung — Strafe geschildert. Im 2. Kriegsjahr (Ms 30-55 [Fig. 48-76]) machen Übergänge über Flüsse, Vormarsch und Vordringen fast die Hälfte der Szenen aus. Dagegen kommt es nirgends zu einer großen Schlacht zwischen zwei Heeren, wie es an der Traians-Säule oft eindringlich geschildert wird. Sondern in aller Regel werden die Gegner einzeln abgeschlachtet und gefangen genommen, die Siedlungen in Brand gesetzt und zerstört (z.B. Ms 7 [Fig. 18-20], 20 [Fig. 39-42], 28 [Fig. 45], 46 [Fig. 66], 63 [Fig. 80], 68 [Fig. 82 und 85] usw.).

Darum wird auf der Marcus-Säule immer wieder, und stärker als bei Traian, der Unterschied zwischen ,guten“ und ,,bösen“ Barbaren hervorgehoben, insbesondere zu Beginn der Feldzüge. Gleich bei den ersten Kampfhandlungen werden zwei hochgestellte Reiter vor den Kaiser geführt, die sich dem allgemeinen Massaker durch Ergebung entzogen haben (Ms 8 [Fig. 21-22]). Beim weiteren Vordringen werden die Römer offenbar von freundlichen Germanen zum Überschreiten eines Flusses aufgefordert (Ms 22). Zu Beginn des 3. Kriegsjahres sucht ein Teil der Barbaren bei den Römern Schutz, während die anderen verfolgt und vernichtet werden (Ms 56 [Fig. 77]). In der Unterscheidung von den kooperativen und willfährigen Fremden sollen Strafe und Brutalität gegen die Rebellen gerechtfertigt erscheinen.

Entsprechend werden die Kriegsfolgen anders als an der Traians-Säule geschildert. Bei Traian gibt es nur nach dem 1. Kriegsjahr eine Szene der harten Rache: Häuser werden angezündet, das Vieh liegt abgeschlachtet am Boden, die waffenfähigen Männer werden umgebracht und die Frauen mit Kleinkindern abgeführt (Ts 30). Später aber wird an der Traians-Säule die Bevölkerung insgesamt zur Umsiedlung gezwungen: eine politische Maßnahme, die zwar einschneidend, aber auf eine neue Ordnung hin orientiert ist (Ts 76, 152/155). An der Marcus-Säule dagegen werden in vielen Szenen die Männer eliminiert, die Frauen in Scharen deportiert, die Herden als Beute davongetrieben (Ms 20 [Fig. 40-42], 43 [Fig. 64], 50 [Fig. 71-73], 61 [Fig. 79], 68 [Fig. 82], 72-73 [Fig. 90], 97 [Fig. 112-114], 102-104 [Fig. 123-124]): als reine Bestrafungen.

\section{Krasse Kontraste}

Dies alles bedeutet, daß der Gegensatz zwischen den Römern und ihren Gegnern an der Marcus-Säule in extremer Weise gesteigert ist.

Bei den Römern werden zwar wenige Bauszenen, aber doch eine Reihe von befestigten Lagern dargestellt (z.B. Ms 6 [Fig. 17], 8 [Fig. 21-22], 29 [Fig. 46-47], 31 [Fig. 49-50], 49-50 [Fig. 68-73], 80 [Fig. 101-103], 101 [Fig. 121], 109 [Fig. 128-129]). Die Gegner dagegen sind völlig schutzlos: Sie besitzen - mit einer einzigen Ausnahme, s.u. - nur Holz-Siedlungen, die von den Römern in Brand gesetzt werden (z.B. Ms 7 [Fig. 18-20], 20 [Fig. 39-42], 43 [Fig. 64], 98 [Fig. 117], 102 [Fig. 123]). Ganz anders die Daker an der Traians-Säule, deren mächtige Befestigungen eine große Herausforderung für die Römer waren (Ts 113-116, 120-122). Marc Aurel muß nicht erobern, sondern nur strafen und rächen.

Ebenso deutlich ist die logistische Überlegenheit der Römer. Vor dem endgültigen Sieg wird der Troß mit Lastwagen eindringlich in Szene gesetzt (Ms 93), wiederum weit deutlicher als an der Traians-Säule (Ts 62/62, 106/7). 
Vor allem aber wird römische Disziplin in krassen Gegensatz zum Chaos der barbarischen Gegner gesetzt. In Szenen des Vormarschs erscheinen die römischen Legionäre in einem stereotypen Gleichschritt, als ob sie schon damals auf dem Exerzierfeld gedrillt worden wären (Ms 67 [Fig. 81], 78 [Fig. 97-98], auch 103, 108 [Fig. 126]). Auch diese Tugend der Disziplin wird vor allem gegen Ende des Krieges in kalkulierter Weise als Voraussetzung des Sieges hervorgehoben. Ähnlich erscheint schon am Ende des 1. Krieges die taktische Disziplin in Form der testudo geschildert (Ms 54 [Fig. 74]); hier ist die einzige gegnerische Festung ins Bild gebracht, sie dient nur als Objekt römischer Kampftechnik. Anschließend wird dieselbe Disziplin in einer besonders geordneten Adlocutio in Szene gesetzt (Ms 55 [Fig. 75-6]).

Dem gegenüber bilden die Germanen nirgends auch nur einen einigermaßen geschlossenen Kampfverband. Auch in dieser Hinsicht hatten die Daker an der Traians-Säule noch ein größeres Potential dargestellt: In den großen Schlachten treten sie mit kollektiver Kraft auf (Ts 24, 32, 66/7, 93, 115/6, 132/3, 134/5). Die Gegner an der Marcus-Säule sind durchweg isolierte Opfer (z.B. Ms 20 [Fig. 39-42], 23, 43, 52, 57, 63 [Fig. 80]).

Die Spitze und höchste Instanz des römischen Heeres ist natürlich der Kaiser: Der ganze Krieg ist eine Demonstration seiner Macht. Das war auch an der Traians-Säule so gewesen; aber wieder ist dies Konzept an der Marcus-Säule in signifikanter Weise gesteigert. In Szenen der Adlocutio wird Marc Aurel aus dem eigenen Heer weit stärker hervorgehoben als Traian: höher und in reiner, symmetrischer Frontansicht (besonders Ms 4 [Fig. 14-15], 9 [Fig. 23], 83 [Fig. 105]) ${ }^{12}$. Das Ritual ist zu einem Bild der Verehrung für die majestätische Erscheinung des Herrschers geworden. Gegenüber den Gegnern aber sind die Unterschiede besonders krass in einer Szene, in der Marc Aurel eine Gesandtschaft empfängt (Ms 49 [Fig. 68-71]): Die Barbaren nähern sich in gekrümmter Haltung, mit verhüllten Armen, wie im Kult der Götter; der Kaiser aber steht auf hohem Podest, symmetrisch umgeben von zwei Offizieren, vor dem Praetorium, dessen Giebel ihn wie ein Tempel überragt!

Dem entspricht es, daß die Gegner an der Marcus-Säule keinen leitenden Herrscher oder Feldherrn haben. Die Daker an der Traianssäule hatten in Dekebalos einen Anführer gehabt, der zwar Aufruhr und Bösartigkeit verkörperte, der aber einen großen, trotzigen und gefährlichen Gegenspieler Traians darstellte (Ts 75, 93, 134/5). Marc Aurel dagegen ist in seiner Majestät allein.

Für einen solchen Kaiser setzen auch die Götter starke Zeichen. Schon an der Traians-Säule war in der ersten großen Schlacht Iuppiter persönlich erschienen, den Blitz schleudernd, in derselben Bewegung wie die römischen Krieger (Ts 24). Es mag sein, läßt sich allerdings nicht beweisen, daß der Gott ein Gewitter verkörpert, das den Römern in der Schlacht zu Hilfe kam, oder einen Blitzschlag, den sie als gutes Omen betrachteten ${ }^{13}$. Jedenfalls wird hier bei der ersten Entscheidungsschlacht des Krieges die Hilfe der Götter versichert, auf die bis zum Ende vertraut wird. Bei Marc Aurel erscheinen solche Zeichen genau zu demselben Zeitpunkt - aber mit weit größerem Eklat. Kurz nach Beginn des 1. Feldzugs wird eine römische Festung von beiden Seiten umzingelt und sogar mit einem Belagerungsturm angegriffen — da schlägt ein

${ }^{12}$ Dazu P.G. HamberG, a.O. (Anm. 3) 141-145.

${ }^{13}$ W. JoBST, 12. Juni 172 n.Chr. Der Tag des Blitz- und Regenwunders im Quadenland. Österreichische Akademie der Wissenschaften, phil.-hist. Klasse, Sitzungsbericht Nr. 335 (Wien 1978). 
Blitz in das Holzgerüst ein und läßt es über den Belagerern zusammenkrachen (Ms 10-11 [Fig. 26-28]). Wenig später wird das römische Heer in einer verzweifelten Situa-tion, von der Trockenheit ausgedörrt und von den Feinden bedrängt, durch einen mirakulösen Regenguß gerettet: für die Römer die Linderung des tödlichen Durstes, für die Gegner eine Überschwemmung, die Menschen und Tiere ins Chaos spült (Ms 16 [Fig. 33-35]). Auch die Götter sind stärker präsent als früher.

\section{Emotionen}

Dies alles bedeutet nicht zuletzt eine Steigerung der emotionalen Effekte. Blitz und Wolkenbruch sind mehr als Götterzeichen: In hoffnungsloser Not sind es wunderbare Rettungen gegen jede Erwartung. Der Zusammenbruch des Belagerungsturmes, die Gier von Menschen und Tieren nach dem rettenden Wasser, die Katastrophe der Überschwemmung: dies alles ist von höchster psychagogischer Kraft. Angst und Entsetzen, Befreiung und Erlösung werden unmittelbar mitgelitten und miterlebt ${ }^{14}$.

Überraschende Details erhöhen die Wirkung. Auf das Dach der testudo werden Schwerter, Wagenräder und ein Topf voll (heißer?) Flüssigkeit hinuntergeworfen, deren Aufschlagen man fast zu hören meint (Ms 54 [Fig. 74]). Später wird die Ankunft eines Boten in einer Festung in Szene gesetzt, in der der Kaiser mit seinen Beratern eindrucksvoll abgeschlossen steht und die geheime Botschaft entgegennimmt (Ms 101 [Fig. 121-122]).

Aber es sind nicht nur die Betrachter der Reliefs, sondern auch die Figuren im Bild selbst, die von stärksten Emotionen ergriffen sind. Mitten in der Not des Verdurstens streckt ein Römer die Hand flehend zum Himmel: Unmittelbar darauf schüttet der Gott des Regens seine rettenden Wasser herab (Ms 16 [Fig. 33-35]) ${ }^{15}$. Bald darauf fleht ein Germane mit derselben Geste die Götter um Rettung vor den Römern an aber umsonst: sein Dorf wird nicht verschont (Ms 20 [Fig. 39-42]).

Emphatische Schilderung von Untergang, Leiden und Emotionen springt an der Marcus-Säule überall in die Augen. Hilflose Feinde am Boden werden ins Gesicht getreten und mit Lanzen niedergestochen; einzelnen Männern stehen Angst und Schrecken im Gesicht (Ms 68 [Fig. 82]) ${ }^{16}$. Vor einem römischen Krieger bricht ein Pferd zusammen, auf dessen Rücken der Reiter bereits tot, rücklings und in verkehrter Richtung ausgestreckt liegt (Ms 24). Tote Gegner hängen gekrümmt im Raum (Ms 50). Sie häufen sich zu Leichenbergen (Ms 89 [Fig. 109]. Ms 109 [Fig. 128]). Besonders widerborstige Germanen werden auf römischen Befehl von ihren eigenen Landsleuten hingerichtet (Ms 61 [Fig. 79]). Verzweifelte Frauen und Kinder werden abgeschleppt (Ms 104 [Fig. 124]).

Gegenüber der Traians-Säule bedeuten derartige Szenen und Motive eine enorme Steigerung der emotionalen Wirkung. Es ist verständlich, daß komplexe ideologische Konzepte von pietas, providentia und consilium als zweitrangig erschienen, wenn die

${ }^{14}$ Hierin sind diese Szenen der sog. ,tragischen“ oder „pathetischen“ Geschichtsschreibung verwandt: T. HöLSCHER, Römische Bildsprache als semantisches System. Abhandlungen der Heidelberger Akademie der Wissenschaften, phil.-hist. Klasse 1987 Nr. 2, 20-33.

${ }^{15}$ Gut zu erkennen bei C. CAPrINo et al., La colonna di Marco Aurelio (Roma 1955) Tav. 16 und Tav. A.

${ }^{16}$ Gute Abbildung bei C. CAPrINO et al., a. O. Tav. O. 
Führung des Krieges in dieser Weise auf Emotionen begründet wurde. Dies ist wohl der Rahmen, in dem auch die emphatischen Gesten der Marcus-Säule gesehen werden sollten.

\section{Stilformen und Mentalität}

Aus dieser Perspektive kann ein Blick auf die Stilformen geworfen werden. Der Stil der Marcus-Säule bedeutet grundsätzlich eine Auflösung der traditionellen klassischen Körperformen, mit dem Ziel eines stärkeren expressiven Ausdrucks. Die Körper erhalten sehr lange Proportionen; sie werden in hohem Maß verformt und verzerrt, weit mehr als der organische Körperbau es zuläßt. Durch eine ähnliche Verformung erhalten die Köpfe einen emphatischen psychischen Ausdruck ${ }^{17}$. Diese Emphase wird noch gesteigert durch zwei allgemeine Prinzipien des Stils: durch die Eliminierung der klassischen Raum-Perspektive, und durch scharfe Kontraste zwischen Licht und Schatten (alle Elemente vereinigt in Ms 68 [fig. 82-85], s. auch 104 [fig. 124]). Der von G.Rodenwaldt beschriebene „Stilwandel“ der spätantoninischen Kunst $^{18}$ dient an der Marcus-Säule dieser emphatischen Steigerung der optischen Wirkung, ganz entsprechend den starken emotionalen und psychagogischen Wirkungen der narrativen Bildmotive.

Daraus ergibt sich die Frage, wie diese Phänomene mit dem spezifischen Thema und der Botschaft der Marcus-Säule und mit dem Erlebnis und den Erfahrungen der Markomannen-Kriege zusammenhängen. Zweifellos wurde das Einbrechen der Germanen bis tief in das römische Reichsgebiet als Choc von größtem Ausmaß empfunden. Fast 300 Jahre lang, seit den Kimbern und Teutonen, war das Imperium nicht mehr angegriffen worden - und nun waren es wieder Germanen, die bis Aquileia vorstießen! Die schriftlichen Zeugnisse lassen noch das Entsetzen ahnen, das sich überall ausbreitete ${ }^{19}$. War es also diese psychologische Hochspannung, in der die Römer mit so ungewöhnlicher Härte reagierten? So daß sie ihre ideologischen Konzepte in den Hintergrund stellten und nur noch auf brutale Gewalt setzten? daß sie die Feinde nicht mehr als mächtige Gegner bekämpften, sondern als Verbrecher hinrichteten? daß sie für diese Botschaft ganz neue emotionale Bildmotive und grelle Stilformen entwickelten?

Sicher hat die psychologische Emphase dieser Reliefs etwas mit der Erfahrung des geschilderten Krieges zu tun: Der Choc wird kompensiert, mit einer emphati-

${ }^{17}$ K. R. KRIERER, Sieg und Niederlage (Wien 1995) 136-157.

${ }^{18}$ Lit. oben Anm. 7.

${ }^{19}$ Quellen: Scriptores Historiae Augustae, Marcus 13,1: Tantus autem terror belli Marcomannici fuit ut undique sacerdotes Antoninus acciverit, peregrinos ritus impleverit, Romam omni genere lustraverit retardatusque a bellica profectione sit. Zum Eindruck der gleichzeitigen Pest in Rom s. Aelius ARISTEIDES, or. 48, 38-45. Allgemein zum Krisenbewußtsein dieser Zeit: G. AlföLdY, Römische Sozialgeschichte (3. Auflage 1984) 133 (mit Literatur). K. Strobel, a.O. (Anm. 7) scheint mir in seiner Gegenposition das Kind mit dem Bad auszuschütten: Es ist natürlich immer möglich, einen globalen (letztlich auf modernen Vorstellungen von umfassenden geschichtlichen Bewegungen beruhenden) Begriff von ,Krise' anzusetzen und dann festzustellen, daß er für die Antike nicht zutrifft. Mit geht es hier nicht um den Begriff, sondern um die Phänomene. 
schen Botschaft. Doch damit kann das Phänomen nicht im vollen Ausmaß erfaßt sein: Kein historisches Ereignis kann eine solche Reaktion ex nihilo, in einer quasi naturgemäßen Kausalität generieren. Manche andere Kriege der Antike waren ein tiefer Choc, ohne daß die Gesellschaft mit solchen Bildern reagiert hätte. So betrachtet, müßte die Botschaft der Marcus-Säule nicht nur eine Reaktion auf einen bestimmten Vorgang der histoire événementelle, sondern darüber hinaus ein Symptom einer allgemeineren und bereits früher angelegten Mentalität der mittleren Kaiserzeit sein.

In der Tat geht die Entwicklung der künstlerischen Stilformen schon vor diesem Krieg in die Richtung zu stärkeren Kontrasten von Hell und Dunkel, zur Auflösung organischer Körperformen und zu stärkeren optischen Effekten ${ }^{20}$. Ein bekanntes Beispiel sind die Reliefs am Sockel der Ehrensäule für Antoninus Pius, kurz nach 161 n.Chr. ${ }^{21}$ : vor Beginn der großen Kriege und mit Themen des Kaiserzeremoniells. Später wird der voll ausgeprägte spätantoninische Stil nicht nur für die Kriege des Marc Aurel und andere kriegerische Themen, etwa auf Schlachtsarkophagen, eingesetzt, sondern auch für die Schilderung verschiedener Mythen, die nichts mit Krieg zu tun haben: Medea, Proserpina und viele andere ${ }^{22}$. Dieser Stil ist also nicht eigens für diese politische Botschaft geschaffen worden: Seine ersten Anfänge liegen früher, und seine Anwendung ist breiter als an der Marcus-Säule.

Offenbar hat die allgemeine, psychologische und mentale Verfassung der römischen Gesellschaft sich langsam, aber stetig in Richtung zu stärkerer emotionaler Reizbarkeit entwickelt ${ }^{23}$. Das hat sicher nichts, wie man oft meint, mit Einflüssen aus dem Orient zu tun. Sondern das Potential zu dieser Entwicklung muß in Rom selbst entstanden sein: Dort war auch das Zentrum dieser Stilformen. Offenbar nahm ganz allgemein die Faszination durch starke psychische Wirkungen zu, durch neue Formen der Religion, fremdartige Rituale, Wundermänner und vieles andere ${ }^{24}$. Ein Symptom ist etwa die steigende Bedeutung des Mithras-Kultes ${ }^{25}$ : In den unterirdischen Versammlungsstätten muß die Beleuchtung durch Fackeln ähnliche Effekte von Licht und Dunkel erzeugt haben, wie sie die Bildkunst anstrebte. Dabei erweckte der Kult zwar einen exotischen Anschein, war aber in Italien entstanden und hatte dort sein Zentrum. Die kollektive Psychologie steigerte sich zu hoher Anspannung. Wahrscheinlich muß man das als Gegenreaktion und Kompensation vor dem Hintergrund der steinernen Stabilität des Reiches in der mittleren Kaiserzeit verstehen.

${ }^{20}$ H. Jung, a.O. (Anm. 7).

${ }^{21}$ L. VoGel, The Column of Antoninus Pius (Cambridge, Mass. 1973) fig. 9-17.

${ }^{22}$ G. KoCH - H. SichtermanN, Römische Sarkophage. Handbuch der Archäologie (München 1982), z.B. Abb. 165 (Eroberung Troias), 181 (Medea), 188 (Meleager), 190 (Niobiden), 197 (Urteil des Paris), 211 (Phaeton).

${ }^{23}$ Das Folgende kann hier nur ganz kurz und unzureichend angedeutet werden. Das Thema wäre eine eingehende Untersuchung unter Einbeziehung der archäologischen Zeugnisse wert.

${ }^{24}$ Dazu A. BIRLEY, Marcus Aurelius (London 1966) 37-38, 202-216. C. P. Jones, Culture and Society in Lucian (London 1986) 133-148.

${ }^{25}$ Zur steigenden Bedeutung des Mithras-Kultes in der zweiten Hälfte des 2. Jahrhunderts n.Chr.: M. Clauss, Mithras, Kult und Mysterien (München 1990) 33-37. Ders., Cultores Mithrae (Stuttgart 1992) 255-258. 
Diesen Prozeß kann man auch an den Erwartungen und Hoffnungen ablesen, die sich auf den Kaiser und das Imperium richteten. Das sensibelste Medium dafür sind die Münzen ${ }^{26 .}$

Seit Beginn der Kaiserzeit war in der offiziellen Ideologie die Ewigkeit des römischen Reiches mit der Macht des Herrschers begründet worden ${ }^{27}$. Bei den Flaviern, die den ersten dynastischen Wechsel plausibel machen mußten, wurde darum Aeternitas auf den Münzen beschworen ${ }^{28}$. Eine Steigerung dieser Vorstellungen findet sich unter Antoninus Pius. Die Zahl der Prägungen, die in irgendeiner Weise auf Aeternitas hinweisen, nimmt stark $\mathrm{zu}^{29}$. Sie sind unmittelbar durch den Tod und die Konsekration der Kaiserin Faustina ausgelöst, schließen aber zugleich in einem weiten Sinn die Ewigkeit des Reiches ein: Einerseits wird Aeternitas mit Attributen vieler anderer Gottheiten präsentiert, andererseits werden andere Gottheiten durch die Beischrift „Aeternitas“ erläutert — oft sind die beiden Verfahren nicht klar voneinander zu unterscheiden. Auf diese Weise erscheint das Konzept der Aeternitas mit Gestalten wie Fortuna, Ceres, Securitas, Spes, Pietas und Providentia verbunden ${ }^{30}$. Alle ideologischen Leitbilder führen auf Aeternitas: Die Ewigkeit der Kaiserherrschaft und des Reiches wird emphatisch und global proklamiert. Gleichzeitig werden neue panegyrische Begriffe für das erreichte oder erhoffte Glück gefunden und auf den Münzen proklamiert: Zu Pax treten Securitas und Tranquillitas, neben Felicitas erscheinen Hilaritas und Laetitia ${ }^{31}$.

${ }^{26} \mathrm{Im}$ Folgenden gebe ich nur die allernotwendigsten Hinweise. Sie können über die Indices von Coins of the Roman Empire in the British Museum I-VI (1923-1975; zitiert im Folgenden: BMC Roman Empire) und The Roman Imperial Coinage I-XI (1923-1994) verifiziert werden. Der Befund würde sich möglicherweise verschieben, wenn die Inschriften systematisch erfaßt würden, was mir hier nicht möglich ist. Die Münzen sind aber, als geschlossene Gattung mit offiziellen ideologischen Botschaften des Staates, auch für sich aussagekräftig.

${ }^{27}$ Zum Konzept der „Ewigkeit“" von Kaiserherrschaft und Reich: M. P. CHARLESWORTH, Providentia and Aeternitas. Harvard Theological Review 29 (1936) 122-132. H. U. INSTINSKY, Kaiser und Ewigkeit. Hermes 77 (1942) 313-355. G. G. BelloNI, „Aeternitas“ e annietamento dei Barbari sulle monete. In: M. SORDI (ed.), I canali della propaganda nel mondo antico (Milano 1976) 220-228. Ders., LIMC I (1981) 244-249 s.v. Aeternitas.

${ }^{28}$ BMC Roman Empire II (1930) 48 Nr. 271-274; 52-53 Nr. 302-304 etc. Index S. 441-442. Danach wurde das Konzept der Aeternitas unter Traian und Hadrian weiter entwickelt, s. BMC Roman Empire III (1936) Index S. 574. F. FERRARIO, Vota publica pro salute felicitate aeternitate Augusti. Annali dell'Istituto Italiano di Numismatica 7-8 (1960-61) 39-57.

${ }^{29}$ BMC Roman Empire IV (1940) Index S. 867 und 910.

${ }^{30}$ BMC Roman Empire IV (1940), vielfach Prägungen für Faustina maior: Fortuna (Ruder, Globus, patera): 43 Nr. 285; 54-56 Nr. 359-372; 240 Nr. 1498-1500; 248 Nr. 1557. - Ceres (Ähren, Fackel): 154 Nr. 1026; 238 Nr. * und 1479; 246 Nr. 1539; 253 Nr. 1586-1587; 489 Nr. 706-708; 651-651 Nr. 1558-1562. - Securitas (Ikonographie nicht sehr spezifisch, z.T. auf Säule gelehnt): 489 Nr. 709-710; 651-652 Nr. 1563-1565. - Spes (zieht Zipfel des Gewands zur Seite, hält z.T. Blume): 241 Nr. *; 247 Nr. 1544-1547; 249 Nr. *. - Pietas (opfernd, velatio): $249 \mathrm{Nr}$. 1558. - Providentia (Globus, Szepter): 43-44 Nr. 286-291; 50 Nr. +; 154 Nr. 1024-1025; $229-$ 230 Nr. 1418-1421; 235 Nr. 1458-1461; 240 Nr. *; 249 Nr. 1559-1560; (Globus, Schleier): 56 Nr. 353-381; 74 Nr. 510; 240 Nr. 1495-1497; 247 Nr. 1548; 396 o.Nr.. Dazu Vesta: 238 Nr. +.

${ }^{31}$ Securitas (seit Nero): LIMC VIII (1997, Supplement) 1090-1093 (U. W. GotTSCHALL). Tranquillitas (seit Hadrian): LIMC VIII (1997) 50-51 (R. VOLLKOMMER). Hilaritas (seit Hadrian): LIMC.VIII (1997, Supplement) 631-633 (Тн. GANSCHOW). Laetitia (seit Antoninus Pius): LIMC VI (1992) 181-185 (H.-J. SCHULZKI). 
Ein weiterer, noch stärkerer Schub setzt unter Commodus und den Severern ein. Schon früher hatten Gottheiten wie Roma, Venus und sogar Annona auf Münzen den Beinamen Felix erhalten; seit Commodus trägt der Kaiser diesen Namen. Entsprechend werden auf Münzen auch zentrale Begriffe der Herrschaft als „felix“ bezeichnet: Victoria, Fortuna, dazu die Concordia des Kaiserpaares ${ }^{32}$. Man jubelt der Prof(ectio) Augg(ustorum) fel(icissima) und dem Adventui Augusti felicissimo $\mathrm{zu}^{33}$. Als neue Gestalten der Glückshoffnungen treten Abundantia, später Uberitas auf $^{34}$.

Die Glückszeit soll ewig dauern: Seit den Severern wird dies zum Ostinato. Der Kaiser selbst und seine Familie werden als Repräsentanten der Aeternitas imperii gerühmt $^{35}$. In der Nachfolge der hadrianischen Roma aeterna erhalten verschiedene politische Leitbegriffe wie: Pax und Concordia, Spes und Securitas, Felicitas und Victoria auf Münzen die emphatische Erklärung ,aeterna“ und „perpetua“ ${ }^{36}$. Neben Tellus stabilis werden Fortuna manens und Perpetuitas selbst beschworen ${ }^{37}$. Nach Felicitas temporum werden auch Laetitia, Hilaritas, Securitas, später Abundantia, Fecunditas und Clementia durch den Zusatz ,temporum“ auf den Zyklus der Zeit bezogen ${ }^{38}$. Felicitas saeculi, Securitas saeculi und das Saeculum frugiferum werden gefeiert $^{39}$.

Neben die zeitliche Ewigkeit tritt die räumliche Unendlichkeit: Schon früher war der Kaiser mit dem Globus als Weltherrscher ausgezeichnet worden; jetzt benennen

${ }^{32}$ Kaiser mit Beinamen Felix: J. BEAUJEU, La religion romaine à l'apogée de l'Empire (Paris 1955) 395-396. P. KNEISSL, Die Siegestitulatur der römischen Kaiser (Göttingen 1969) 116, 159160. - Venus: seit Hadrian. - Roma: Hadrian, Commodus.- Annona: Antoninus Pius. - Fortuna: seit Commodus. - Victoria: Commodus, Septimius Severus / Caracalla. - Concordia: seit Septimius Severus.

${ }^{33}$ BMC Roman Empire V (1950) 45-46 Nr. 150-156; 147 Nr. 595-598; 149 Nr. 607; 177 Nr. 134-135; $193 \mathrm{Nr}$. +; $508 \mathrm{Nr} .1$.

${ }^{34}$ Abundantia (gesichert seit Elagabal): LIMC I (1981) 7-10 (R. FonTAN BARREIRO). Uberitas (seit Traianus Decius): LIMC VIII (1997) 157-158 (R. VOLLKOMMER).

${ }^{35}$ BMC Roman Empire V (1950) 156-158 Nr. 1-6; 189 unten; 191 Nr. 184; $213-214$ Nr. $307-$ 308; 230 Nr. +; 379 Nr. 124-125.

${ }^{36}$ Aeternus/-a: Roma: seit Hadrian. Pax: seit Marc Aurel. Concordia: Septimius Severus, Tetrarchen. Victoria: seit Septimius Severus. Felicitas: Gallienus. Vesta: Gallienus. Perpetuus/-a: Roma: Vespasian. Felicitas: Commodus, Alexander Severus. Concordia: Septimius Severus. Spes: seit Septimius Severus. Securitas: seit Septimius Severus. Pax: Vorläufer Claudius (!), dann Tacitus. Victoria: seit Tacitus. S. auch Potestas (?): Severus Alexander.

${ }^{37}$ Fortuna Manens (Commodus): BMC Roman Empire IV (1940) 851 Nr. § mit S. 858. Tellus stabilis (Hadrian, Septimius Severus / Caracalla): BMC Roman Empire III (1936) 332334 Nr. 737-749; 362 (j); 477 Nr. 1565; 486-487 Nr. 1625-1627. BMC Roman Empire V (1950) 317 Nr. §. - Perpetuitas (Alexander Severus): BMC Roman Empire VI (1962) 159 Nr. 472; 161-162 Nr. 499-502; s. auch 164 Nr. 534.

${ }^{38}$ Felicitas temporum: seit Hadrian (?) oder Antoninus Pius. - Laetitia temporum: seit Pertinax und Septimius Severus. - Hilaritas temporum: Didius Iulianus. - Securitas temporum: seit Septimius Severus / Caracalla. - Abundantia temporum: Severus Alexander. - Fecunditas temporum: Severus Alexander, Philippus Arabs. - Clementia temporum: seit Gallienus.

${ }^{39}$ Felicitas saeculi (bzw. saeculi Felicitas): seit Antoninus Pius. - Securitas saeculi: seit Elagabal. - Saeculum frugiferum: seit Pertinax und Septimius Severus. 
ihn Münzlegenden explizit als Rector orbis ${ }^{40}$. Entsprechend weltweit sind die Segnungen seiner Herrschaft: Securitas orbis, später Spes Felicitatis orbis ${ }^{41}$. Diese ganze unendliche Segenszeit wird von dem Kaiser garantiert, der jetzt in Inschriften das Epitheton ,invictus“ erhält ${ }^{42}$; ebenso wie auf Münzen Iuppiter und Mars ,,invictus“, Virtus und Fortitudo ,invicta“ genannt werden ${ }^{43}$. Später, im 3. Jahrhundert, wird der Kaiser victor ubique, victor undique, victor omnium gentium, victoriosus semper. Seine Herrschaft sichert ubique $\operatorname{pax}^{44}$.

Und die Ubiquität betrifft nicht nur den Herrscher. Auf Sarkophagen werden die verschiedensten Mythen: der Raub der Proserpina, Selene und Endymion, der Sturz des Phaeton, Mars und Rhea Silvia, dazu die Personifikationen der Jahreszeiten, in ein kosmisches Feld zwischen Tellus und Okeanos gestellt ${ }^{45}$.

Das höchste Glück, immer und überall: Dieser emphatische Superlativismus ist die psychologische Gegenseite zu der emotionalen Anspannung, die die Kriegsszenen der Marcus-Säule beherrscht.

\section{Schlu $\beta$}

Die kurze Skizze mag deutlich machen, wie es zu dem psychagogischen Ausbruch der Reliefs an der Marcus-Säule kommen konnte. Die versteinerte Sicherheit der hohen Kaiserzeit scheint im Lauf der Jahrzehnte ein kompensatorisches Maß an kollektiver psychologischer Reizbarkeit erzeugt zu haben. Als dann der unerwartete Einbruch der Nordvölker kam, reagierte man einerseits mit der Botschaft schierer Strafe und Brutalität, andererseits mit der exzessiven Beschwörung globaler Glücksvisionen. Beides bezeugt einen allgemeinen emotionalen Hitzegrad, der weiterer Erforschung bedarf.

\footnotetext{
${ }^{40}$ Kaiser mit Globus: T. HÖLSCHER, Victoria Romana (Mainz 1967) 22-34. Rector orbis auf Münzen: seit Pertinax, Didius Iulianus und Septimius Severus. Spätere Steigerung: Restitutor orbis: seit Valerian. Conservator orbis: seit Gallienus. Pacator orbis: seit Aurelian. Defensor Orbis: Victorinus. Restitutor totius orbis: Constantin.

${ }^{41}$ Securitas orbis: seit Commodus. - Spes Felicitatis orbis: Philippus Arabs.

${ }^{42}$ M. IMHOF, Invictus. Museum Helveticum 14 (1957) 197-215. St.WeInSTOCK, Victor and Invictus. Harvard Theological Review 50 (1957) 211-247.

${ }^{43}$ Iuppiter invictus: Septimius Severus. - Mars invictus: Pescennius Niger, Aurelian. Invicta Virtus: Septimius Severus. - Fortitudo invicta: Clodius Albinus.

${ }^{44}$ T. HÖlscher, a.O. (Anm. 38) 167. Vgl. P. KNEISSL, a.O. (Anm. 31) 224-225. Beispiele solcher Legenden lassen sich vermehren.

${ }^{45}$ Belege bei G. Koch - H. Sichtermann, a.O. (Anm. 21) in den Abschnitten zu den betreffenden Themen. Dazu eine unveröffentlichte Magister-Arbeit von Barbara ReIMLING, Tellus und Okeanos auf römischen Sarkophagen (Heidelberg 1992).
} 